\title{
Linezolid-related adverse effects in clinical practice in children
}

\author{
Nuri Bayram, M.D., Associate professor ${ }^{a}$, Mine Düzgöl, M.D. ${ }^{a}$, Ahu Kara, M.D. ${ }^{a}$, \\ Fatih M. Özdemir, M.D. ${ }^{b}$ and Ilker Devrim, M.D., Associate professor ${ }^{a}$
}

\begin{abstract}
Introduction: Linezolid may cause adverse effects such as thrombocytopenia, which were found to be dependent on receiving linezolid for longer than 2 weeks. There are limited studies concerning the safety and timing of linezolidrelated adverse effects in children. Objective of this study was to evaluate the incidence of adverse effects associated with linezolid, with especially focusing on the time of occurrence. Population and Methods: All children ( $\leq 18$ years of age) who received $\geq 3$ days of linezolid therapy were included in this study. Adverse effects attributed to linezolid and time of occurrence of side effects was evaluated.

Results: A total of 179 children were enrolled to the study. The patients' median age was 4 years (6 days to 17 years). During linezolid treatment, $36(20.1 \%)$ patients experienced adverse effects. The most common adverse effect was thrombocytopenia that was detected in 26 patients $(14.5 \%)$. Other adverse effects were as following; elevated liver enzymes in 4 patients, leucopenia and anemia in 2 patients, renal function impairment in one patient, and serious skin reactions in 3 patients. Adverse effects were detected within median 7.5 days of therapy (ranging from 4 to 18 days). Among 36 patients, $26(72.2 \%)$ patients had adverse effect on the first 10 days of therapy.

Conclusions: Transient adverse effects were detected in $20.1 \%$ of the patients during linezolid therapy. These adverse effects may be detected earlier than ten days of treatment. Linezolid should be prescribed safely in children with monitoring adverse effects especially platelet count and level of liver enzymes.

Key words: Linezolid, adverse effects, child, thrombocytopenia.
\end{abstract}

http:/ / dx.doi.org/10.5546/ aap.2017.eng.470

Children's Hospital İzmir, Turkey.

b. Dr. BehçetUz

Children's Hospital, İzmir, Turkey.

E-mail address:

Nuri Bayram, M.D.:

nuribayram@gmail.com

To cite: Bayram N, Düzgöl M, Kara A, et al. Linezolidrelated adverse effects in clinical practice in children. Arch Argent Pediatr 2017;115(5):470-475.

\section{INTRODUCTION}

Linezolid is the first antibiotic in the oxazolidinone class to be approved and used in children, including premature neonates. ${ }^{1,2}$ It has an excellent activity against most methicillin-sensitive and methicillin-resistant Grampositive bacteria. ${ }^{3}$ Linezolid was originally approved by Food and Drug Administration of United States for pediatric use in 2002, for treatment indicated infections, such as skin and skin structure infections, communityacquired and nosocomial pneumonia, and infections with vancomycinresistant Enterococcus faecium. ${ }^{4}$ The advantages of linezolid compared to glycopeptide antibiotics include clinical and microbiological efficacy, excellent tissue penetration and lack of requirement for therapeutic drug monitoring. ${ }^{5}$ It has a low adverse effect profile compared with glycopeptide antibiotics. However, linezolid has been reported to cause serious adverse effects such as neuropathy, elevated liver enzymes, and myelosuppresion, including thrombocytopenia and leucopenia. ${ }^{6}$

Data on the adverse effects of linezolid is mainly based on adult reports. Currently, there are limited studies concerning the safety of linezolid in pediatric patients. Therefore, the objective of this study was to evaluate the incidence of adverse effects associated with linezolid, with especially focusing on the time of occurrence.

\section{MATERIALS AND METHODS}

This retrospective study was conducted between June 2010 to June 2015 at Dr. BehçetUz Children's Hospital, a referral center for pediatric infectious diseases in the Aegean 
Region of Turkey. All children ( $\leq 18$ years of age) who received $\geq 3$ days of linezolid therapy as a part of suspected or proven Gram-positive infections were included in the study. Any patient with an abnormal platelet count $\left(<150 \times 10^{9} / \mathrm{L}\right)$ prior to therapy was excluded.

Data of the patients was collected from medical records including information on demographic characteristics (age, gender, prematurity, medical history); underlying diseases or co-morbidities; length of hospital stay; type of microbiological and infection data; dosage and duration of linezolid therapy; outcome of linezolid therapy.

Linezolid was administered intravenously at a dosage of $10 \mathrm{mg} / \mathrm{kg}$ three times daily in children aged 0 to 11 years and $10 \mathrm{mg} / \mathrm{kg}$ (maximum dose, $600 \mathrm{mg}$ ) twice daily in older children.

The laboratory findings such as hematologic properties (white blood cell count, hemoglobin value, platelet count), routine biochemical tests, hepatic and renal functions were recorded from medical records. Side effects attributed to linezolid were also evaluated. Adverse effects were defined as events which occurred during linezolid use but were unexplainable by the natural clinical course of the underlying diseases or other drugs. For this purpose, hematological effects included leucopenia, thrombocytopenia, anemia; hepatic impairment included hyperbilirubinemia, elevation in hepatic transaminase levels (alanine aminotransferase elevation more than 3 times the upper limit of normal); gastrointestinal effects included nausea, vomiting, pancreatitis, and diarrhea; nephrotoxicity included acute renal impairment (rise in creatinine of $\geq 50 \%$ from its baseline value and / or a fall in the glomerular filtration rate by $\geq 25 \%$, and / or a decrease in urine output below $0.5 \mathrm{ml} / \mathrm{kg} / \mathrm{h}$ for 6 hour or more); skin effects included rash and itching; and neurological impairment were all evaluated. Thrombocytopenia was defined as either a platelet count of $<150 \times 10^{9} / \mathrm{L}$ or $50 \%$ reduction in platelet count from baseline level at initiation of linezolid therapy. ${ }^{7}$

Outcomes of the patients based on the response to linezolid treatment were defined as cure, improved or failed. Cure was defined as the resolution of clinical and laboratory findings of infection for which linezolid were administered. Persistence or progression of the signs of infection or lack of microbiological eradication indicated failure outcome. Improved course was defined as any situation between cure and failure outcome. Mortality was defined as deaths during treatment and follow-up period.

Statistical analysis was analyzed using SPSS software version 20.0 (IBM Corp., Somers, NY). Categorical variables were analyzed using a chisquare or Fisher exact test. Continuous variables were analyzed using Student's $t$ test or the MannWhitney $U$ test. A $P$ value of $<0.05$ denoted statistical significance.

\section{RESULTS}

During the study period, data of 211 linezolid treatment courses were evaluated. Of these, 24 patients were excluded due to the inadequate data. Eight patients who had died on the first

TABLE 1. Clinical and demographic characteristics of 179 patients enrolled to the study

\begin{tabular}{lc}
\hline Characteristics and underlying medical conditions & Value \\
\hline Number of patients & 179 \\
Age (years), median & 4 (6 days - 17 years) \\
Prematurity & \\
$\quad$ Gestational age $<28$ weeks, N $(\%)$ & $3(1.6)$ \\
$\quad$ Gestational age $28-32$ weeks, N $(\%)$ & $8(4.4)$ \\
$\quad$ Gestational age $>32-36$ weeks, N (\%) & $5(2.7)$ \\
Male, N $(\%)$ & $106(59.2)$ \\
Treatment duration, days (range) & $13(4-31$ days) \\
Patients' admitted unit & \\
$\quad$ Pediatric intensive care unit, N $(\%)$ & $69(38.5)$ \\
$\quad$ Hematology - Oncology unit, N $(\%)$ & $68(37.9)$ \\
$\quad$ Neonatal intensive unit, N $(\%)$ & $21(11.7)$ \\
General pediatric unit, N (\%) & $12(6.7)$ \\
$\quad$ Pediatric intensive surgery care unit, N $(\%)$ & $9(5.5)$ \\
Outcome, & \\
$\quad$ Cured, N $(\%)$ & $128(71.5)$ \\
$\quad$ Clinical improvement, N $(\%)$ & $33(18.4)$ \\
$\quad$ Mortality, N $(\%)$ & $18(10.0)$ \\
\hline
\end{tabular}


three days of linezolid treatment were also excluded. A total of 179 children were enrolled to the study. The patients' median age at admission was 4 years (6 days to 17 years), of whom $106(59.2 \%)$ were males and $73(40.8 \%)$ were females. Sixteen patients were preterm infants; $3(1.6 \%)$ of them born with $<28$ weeks' gestational age, $8(4.4 \%)$ with $28-32$ weeks, and $5(2.7 \%)$ of them with $32-36$ weeks' gestational age. The median duration of linezolid therapy was 12 days (ranging from 4 to 31 days). Baseline characteristics are listed in Table 1.

Among the treatment courses, 69 (38.5\%) were administered to patients in pediatric intensive care unit, $68(37.9 \%)$ to pediatric hematologyoncology patients, $21(11.7 \%)$ to neonatal patients, $12(6.7 \%)$ to patients with immunodeficiency, and the remaining $9(5.5 \%)$ to patients in pediatric surgery intensive care unit. Table 2 shows the number and proportions of the patients with underlying diseases and co-morbidities.

In all children linezolid was administered as second-line treatment except the patients that vancomycin resistant Enterococcus (VRE) or vancomycin resistant Staphylococci were documented as pathogen. Linezolid was mainly administered due to the clinical failure of the patients while receiving glycopeptide therapy ( $85 / 179$ cases, $47.4 \%)$. Target based therapy according to the microbiologically documented diagnosis were administered in $94(52.5 \%)$ children. Of these patients, the most commonly isolated pathogen was coagulase negative Staphylococci (53 cases, 29.6\%), followed by VRE (39 cases, $21.7 \%$ ), and methicillin resistant Staphylococcus aureus (2 cases, 1.1\%). All isolates were susceptible to linezolid (MIC $\leq 2 \mathrm{mg} / \mathrm{L}$ ) according to the Clinical and Laboratories Standards Institute (CLSI) guidelines. ${ }^{8}$

The sites of infections which clinically and microbiologically confirmed were as follows; in 134 patients $(74.9 \%)$ bloodstream infections, in 19 patients $(10.6 \%)$ urinary tract infections, in 10 patients $(5.6 \%)$ central nervous infections, in 9 patients $(5.0 \%)$ ventilator associated pneumonia, in 6 patients $(3.3 \%)$ soft tissue abscess, and in one patient peritonitis.

During linezolid treatment, $36(20.1 \%)$ patients experienced adverse effects. The main adverse effect of linezolid therapy was thrombocytopenia. Linezolid-induced thrombocytopenia was detected in 26 patients $(14.5 \%)$. Final platelet counts of $<50 \times 10^{9} / \mathrm{L}$ were detected in 11 patients $(6.1 \%)$, of whom two patients required thrombocyte transfusions. Platelet count decreases of more than $50 \%$ of initial count with the count of $<150 \times 10^{9} / \mathrm{L}$ was determined in remainder 15 patients $(8.3 \%)$. Leucopenia and anemia was found in $2(1.1 \%)$ patients. We did not observe neutropenia in cases during therapy.

The other adverse effects were as following; transiently elevated liver enzymes in $4(2.2 \%)$ patients, renal function impairment in one patient, and serious skin reactions with exanthema and itching were found in $3(1.6 \%)$ patients. All of these abnormalities were reversible. Adverse effects associated with linezolid therapy were listed in Table 3.

TABLE 2. Clinical indications of linezolid therapy for 179 patients

\begin{tabular}{lcc}
\hline Site of infections & Number of patients & Percentage (\%) \\
\hline Bloodstream infection & 134 & 74.9 \\
Urinary tract infection & 19 & 10.6 \\
Central nervous infection & 10 & 5.6 \\
Ventilator associated pneumonia & 9 & 5.0 \\
Soft tissue infection & 6 & 3.3 \\
Peritonitis & 1 & 0.6 \\
Total & 179 & 100 \\
\hline
\end{tabular}

TABLE 3. Adverse effects associated with linezolid therapy (36/179 patients, 20.1\%)

\begin{tabular}{lcc}
\hline Adverse effect & Number of patients & Percentage (\%) \\
\hline Thrombocytopenia & 26 & 14.5 \\
Leucopenia and anemia & 2 & 1.1 \\
Elevated liver enzymes & 4 & 2.2 \\
Renal function impairment & 1 & 0.6 \\
Skin reactions & 3 & 1.6 \\
\hline
\end{tabular}


One hundred twenty-eight patients $(71.5 \%)$ were considered as to be cured and 33 patients $(18.4 \%)$ had clinical improvement. Mortality rates were found as $10.0 \%$ (18/179 patients) during the therapy due to the severe septic shock and respiratory failure mostly attributed to the underlying diseases.

\section{The correlation of adverse effects with duration of linezolid}

The median duration of linezolid therapy was 13 days (ranging from 4 to 31 days) in children with no adverse effects with linezolid group, and 12 days (ranging from 5 to 28 days) in children with adverse effects, and no significant difference was present between these two groups by means of linezolid duration $(p=0,297)$. The patients under linezolid therapy had experienced the adverse effects with a median of 7.5 days of therapy (ranging from 4 to 18 days). Among 36 patients, $26(72.2 \%)$ patients had adverse effect on the first 10 days, while the remaining $(27.8 \%)$ had adverse effects after the first 10 days of therapy (Data shown in Figure 1). Thrombocytopenia which was the most common adverse effect was observed in $17(77.2 \%)$ patients on the first period, while in $5(22.7 \%)$ of the patients thrombocytopenia had developed after the first 10 days of therapy.

\section{DISCUSSION}

Linezolid has antibacterial activity against Gram-positive bacteria. The safety and efficacy of linezolid in adults have been evaluated in large clinical trials. This report includes one of the largest pediatric populations who received linezolid therapy. The current study points out that adverse effects associated with linezolid are not common and may be detected in the earlier course of the therapy.

Most of the study population received linezolid as the target based therapy according to the microbiologically documented diagnosis and the overall outcomes were favorable, with high cure rates and improved courses in $90 \%$ of patients. This success rates are similar with the study conducted by Garazzino et al. ${ }^{9}$ Although linezolid is generally well tolerated, some concerns have been raised regarding hematologic toxicity, including neutropenia and thrombocytopenia, which was found to be dependent on dose and duration of therapy. ${ }^{10}$ Thrombocytopenia is a common adverse effect in adult patients and the incidence of linezolidrelated thrombocytopenia has been reported about $15-50 \% .7,11,12$ The other common drugrelated side effects reported with linezolid therapy were diarrhea, nausea, headache, neuropathy,

FIGURE 1. Cumulative adverse effects through the linezolid treatment days, most patients accumulated in the first 10 days period of the linezolid treatment $(77.2 \%$ versus $22.78 \%)$

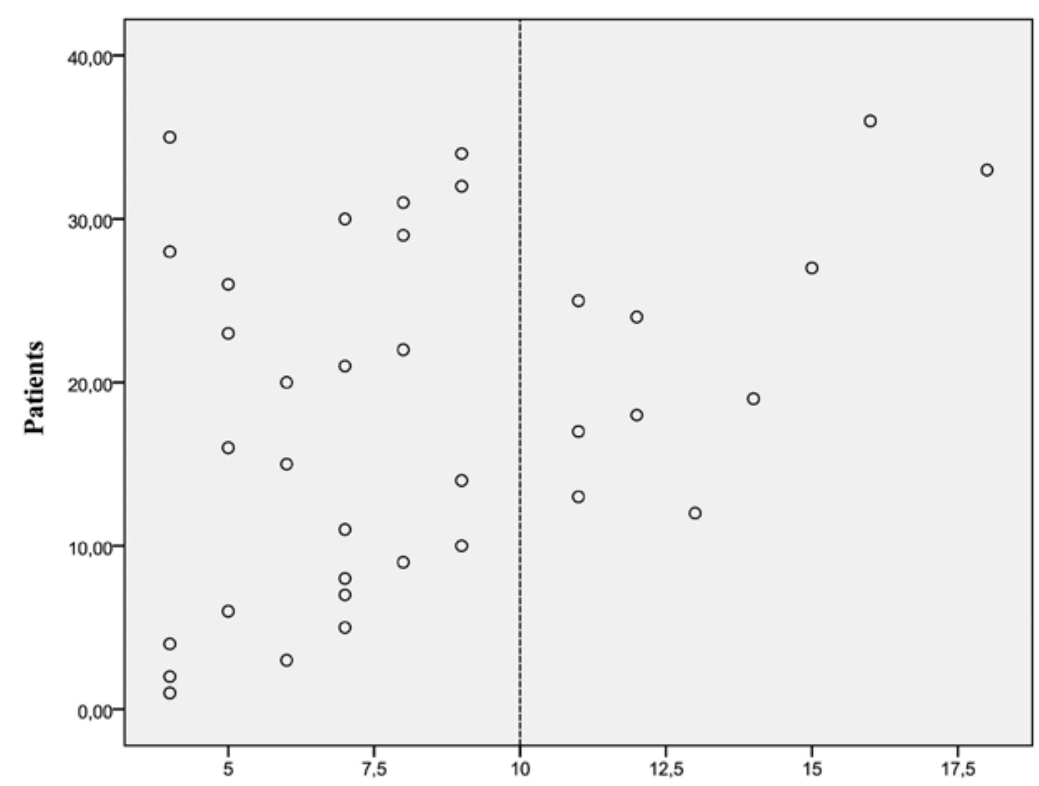

Duration of treatment, days 
and abnormal liver function tests. ${ }^{13}$ These adverse effects were mostly detected in patients receiving linezolid for longer than 2 weeks. ${ }^{13}$

Despite its increasing use, clinical data with linezolid in the pediatric population is limited. In some pediatric studies, $18.8 \%$ to $25.6 \%$ of children were documented to have experienced adverse effects associated with linezolid therapy, most notably liver function alterations, hematological and gastrointestinal disturbances. ${ }^{9}$ In the current study, thrombocytopenia was the most frequent adverse effect associated with linezolid compatible with literature. Although this adverse effect were usually mild and reversible, two of the thrombocytopenia detected patients required platelet infusion, thus close monitoring of hematological parameters is crucial. Reversible elevated liver enzymes, skin reactions, leucopenia, anemia and renal function impairment were the other adverse effects detected in our population. Contrary to the literature, none of the patients had neutropenia, or neuropathy which was also been associated with the children treated with linezolid. ${ }^{14,15}$ Adverse effects associated with gastrointestinal disturbances were also not detected in this study, probably of using only intravenous formula of linezolid.

In adults, the long-term use of linezolid has been reported to be associated with adverse effects, especially thrombocytopenia owing to myelosuppression. ${ }^{5}$ In general, adverse effects are reported less in linezolid treated children than adults, possibly due to reduced susceptibility of children to mitochondrial toxicity, and fewer cases of long-term treatment. ${ }^{9,16}$ The exact mechanism of thrombocytopenia is unknown, but an immune-mediated phenomenon has been proposed. ${ }^{6}$ Data from clinical trials suggest that myelosuppression is associated with longterm therapy. ${ }^{17}$ Thrombocytopenia and a slight increased risk for anemia were evident in patients who had linezolid treatment exceeding 2 weeks' duration. ${ }^{5}$ Studies in adults suggest a slight risk of thrombocytopenia that is increased with longer duration of linezolid, but reversible with drug cessation. ${ }^{18}$ However, pediatric data from clinical trials of shorter duration of linezolid therapy may also cause mild reversible thrombocytopenia in children. ${ }^{19}$ Opposite to the most of data, most of the patients in the present study had experienced the adverse effects within the first ten days of therapy. Thrombocytopenia was the most common adverse effect on the first 10 -days period. Similarly, shorter duration of courses associated with adverse effects, especially thrombocytopenia, were also reported. Zhang et al. reported that the duration of 7 days of linezolid therapy had been associated with thrombocytopenia and concluded that shorter therapies than two weeks may also cause adverse effects. ${ }^{18}$ Orrick et al. proposed that the risk of thrombocytopenia may occur earlier than the 14-day course of linezolid. ${ }^{20}$ In a study including 108 pediatric patients also revealed that the duration of linezolid treatment did not correlate with the adverse effects. ${ }^{14}$ Lactic acidosis is an uncommon adverse effect of linezolid therapy that occurs in adult patients with longer duration of linezolid. ${ }^{21}$ However, linezolid-related lactic acidosis in children tends to develop earlier than adults that in two reports, lactic acidosis had been detected on the first two days of linezolid administrations. ${ }^{22,23}$ Thus, it can be assumed that adverse effects in children may occur earlier than adults due to differences in pharmacokinetics and toxicodynamics of linezolid in pediatric population.

In the present study, $21(11.7 \%)$ patients were newborn infants, and of these, 16 patients were preterm neonates. Transient adverse effects were detected in only three patients; while two patients were experiencing thrombocytopenia; one patient had elevated liver enzymes. The linezolid therapy was safe and effective in the neonatal period similar with the literature., ${ }^{2,14}$

Several considerations should be noted when interpreting the results. Firstly, this was a retrospective study, which has inherent limitations when compared to randomized clinical trials. Secondly, this study includes all children with different underlying diseases and co-morbidities. Third, some effects such as neuropathy are so difficult to identify in pediatric population, so that the observational adverse effects might be undetected. However, we must emphasize that the present study includes one of the largest pediatric population treated with linezolid. Based on data, linezolid should be prescribed safely in pediatric patients with monitoring adverse effects especially platelet count and level of liver enzymes.

In conclusion, in the present study transient adverse effect were detected in $20.1 \%$ of the patients during linezolid therapy. Adverse effects may be detected earlier than ten days of treatment. Use of linezolid in children must be evaluated by prospective and randomized controlled studies to support our data. 


\section{REFERENCES}

1. Chiappini E, Conti C, Galli L, et al. Clinical efficacy and tolerability of linezolid in pediatric patients: a systematic review. ClinTher 2010;32(1):66-88.

2. Kocher S, Muller W, Resch B. Linezolid treatment of nosocomial bacterial infection with multiresistant Grampositive pathogens in preterm infants: asystematic review. Int J Antimicrob Agents 2010;36(2):106-10.

3. Gostelow M, Gonzalez D, Smith PB, et al. Pharmacokinetics and safety of recently approved drugs used to treat methicillin-resistant Staphylococcus aureus infections in infants, children and adults. Expert Rev Clin Pharmacol 2014;7(3):327-40.

4. Dotis J, Iosifidis E, Ioannidou M, et al. Use of linezolid in pediatrics: acritical review. Int J Infect Dis 2010;14(8): e638-48.

5. Bassetti M, Baguneid M, Bouza E, et al. European perspective and update on the management of complicated skin and soft tissue infections due to methicillin-resistant Staphylococcus aureus after more than 10 years of experience with linezolid. Clin Microbiol Infect 2014;20 Suppl 4:3-18.

6. Vinh DC, Rubinstein E. Linezolid: a review of safety and tolerability. J Infect 2009;59 Suppl 1:S59-74.

7. Natsumoto B, Yokota K, Omata F, et al. Risk factors for linezolid-associated thrombocytopenia in adult patients. Infection2014;42(6):1007-12.

8. Tenover FC, Moellering RC Jr. The rationale for revising the Clinical and Laboratory Standards Institute vancomycin minimal inhibitory concentration interpretive criteria for Staphylococcus aureus. Clin Infect Dis 2007;44:1208-15.

9. Garazzino S, Krzysztofiak A, Esposito S, et al. Use of linezolid in infants and children: a retrospective multicentre study of the Italian Society for Paediatric Infectious Diseases. J Antimicrob Chemother 2011;66(10):2393-7.

10. Bradley JS, Sauberan JB. Antimicrobial agents. In: Long SS, Pickering LK, Prober CG, eds. Principles and Practice of Pediatric Infectious Diseases. 4th ed. New York: Elsevier Saunders; 2012:1453-84.

11. Chen C, Guo DH, Cao X, et al. Risk factors for thrombocytopenia in adult Chinese patients receiving linezolid therapy. Curr Ther Res Clin Exp 2012;73(6):195-206.
12. Takahashi $Y$, Takesue $Y$, Nakajima $K$, et al. Risk factors associated with the development of thrombocytopenia in patients who received linezolid therapy. J Infect Chemother 2011;17(3):382-7.

13. Rodvold KA, McConeghy KW. Methicillin-resistant Staphylococcus aureus therapy: past, present, and future. Clin Infect Dis 2014;58 Suppl 1:S20-7.

14. Simon A, Müllenborn E, Prelog M, et al. Use of linezolid in neonatal and pediatric inpatient facilities: results of a retrospective multicenter survey. Eur J Clin Microbiol Infect Dis 2012;31(7):1435-42.

15. Nambiar S, Rellosa N, Wassel RT, et al. Linezolidassociated peripheral and optic neuropathy in children. Pediatrics 2011;127(6):1528-32.

16. Garcia-Prats AJ, Rose PC, Hesseling AC, et al. Linezolid for the treatment of drug-resistant tuberculosis in children: A review and recommendations. Tuberculosis (Edinb) 2014;94(2):93-104.

17. Gerson SL, Kaplan SL, Bruss JB, et al. Hematologic effects of linezolid: summary of clinical experience. Antimicrob Agents Chemother 2002;46(8):2723-6.

18. Zhang Z, Liang Z, Li H, et al. Comparative evaluation of thrombocytopenia in adult patients receiving linezolid or glycopeptides in a respiratory intebsive care unit. Exp Ther Med 2014;7(2):501-7.

19. Meissner HC, Townsend T, Wenman W, et al. Hematologic effects of linezolid in young children. Pediatr Infect Dis J 2003;22(9):S186-92.

20. Orrick JJ, Johns T, Janelle J, et al. Thrombocytopenia secondary to linezolid administration: what is the risk? Clin Infect Dis 2002;35(3):348-9.

21. Boutoille D, Grossi O, Depatureaux A, et al. Fatal lactic acidosis after prolonged linezolid exposure for treatment of multidrug-resistant tuberculosis. Eur J Intern Med 2009;20(6):e134-5.

22. Ozkaya-Parlakay A, Kara A, Celik M, et al. Early lactic acidosis associated with linezolid therapy in paediatric patients. Int J Antimicrob Agents 2014;44(4):334-6.

23. Su E, Crowley K, Carcillo JA, et al. Linezolid and lactic acidosis: a rolefor lactatemonitoring with long-term linezolid use in children. Pediatr Infect Dis J 2011;30(9):804-6. 